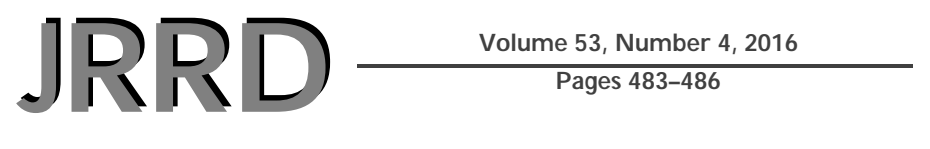

\title{
Case study: Gluteal compartment syndrome as a cause of lumbosacral radiculoplexopathy and complex regional pain syndrome
}

\author{
Andrew Lederman, MD; ${ }^{1-2 *}$ David Turk, MD ${ }^{1-2}$ Antonio Howard, MD $;^{1-2}$ Srinivas Reddy, MD; ${ }^{3}$ Michelle \\ Stern, $\mathrm{MD}^{2}$ \\ ${ }^{1}$ Department of Rehabilitation Medicine, Montefiore Medical Center, Bronx, NY; Departments of ${ }^{2}$ Rehabilitation Medicine \\ and ${ }^{3}$ Surgery, Jacobi Medical Center, Bronx, NY
}

\begin{abstract}
We present the case of a 24 yr old male who was diagnosed with gluteal compartment syndrome and was subsequently found to have developed lumbosacral radiculoplexopathy and complex regional pain syndrome. The patient's gluteal compartment syndrome was diagnosed within $24 \mathrm{~h}$ of presentation to the emergency room, and he underwent emergent compartment release. While recovering postoperatively, persistent weakness was noted in the right lower limb. Results of electrodiagnostic testing were consistent with a lumbosacral radiculoplexopathy. After admission to inpatient rehabilitation, the patient complained of pain, burning sensation, and numbness in the distal right lower limb. Based on clinical findings, he was diagnosed with complex regional pain syndrome type II, or causalgia, and was referred for a lumbar sympathetic block under fluoroscopic guidance. Sympathetic block resulted in relief of the patient's symptoms. He was discharged home with good pain control on oral medications.
\end{abstract}

Key words: case report, causalgia, complex regional pain syndrome, electromyography, fasciotomy, gluteal compartment syndrome, lumbosacral radiculoplexopathy, renal failure, rhabdomyolysis, sympathetic block.

\section{INTRODUCTION}

Complex regional pain syndrome (CRPS) is reported in most retrospective series to have a prevalence of less than 2 percent and an incidence of around 5 cases per 100,000 persons per year [1]. CRPS affecting the lower limbs accounts for about one-third of cases and is thus quite rare. Its rarity undoubtedly contributes to the challenges in recognizing, diagnosing, and treating this very disabling condition.

Gluteal compartment syndrome (CS) is similarly uncommon. It has been reported after prolonged immobility due to sedation for surgery or drug intoxication. The term CS refers to a severe pathologic state resulting from sudden or chronic abnormal rise in muscle compartment pressure, leading to a loss of circulation and resultant tissue necrosis. In many cases of CRPS, there is an antecedent history of trauma and/or nerve compression. We present a case of an acute gluteal CS that preceded the development of CRPS in the same lower limb.

The following case presentation serves to help care providers recognize, understand, and be familiar with CS and CRPS. Additionally, by outlining the patient's course from emergency room (ER), operating room, intensive care unit, acute rehabilitation, and ultimately to discharge, this case report illustrates the wide array of opportunities a rehabilitation physician has to contribute

\footnotetext{
Abbreviations: CRPS = complex regional pain syndrome, CS = compartment syndrome, ER = emergency room.

*Address all correspondence to Andrew Lederman, MD; Department of Rehabilitation Medicine, Montefiore Medical Center, 150 E. 210th St, Bronx, NY 10467; 718-9204083; fax: 718-920-4133. Email: alederma@montefiore.org http://dx.doi.org/10.1682/JRRD.2015.01.0007
} 
to the diagnosis, treatment, and outcome for the acute trauma patient.

\section{CASE PRESENTATION}

Acute CS is a surgical emergency that can result in ischemia, limb loss, and possibly death [2-3]. In this case, a $24 \mathrm{yr}$ old African-American male with a past medical history of smoking and illicit drug use presented to the ER of a level 1 trauma center with complaints of right buttock pain and profound right leg weakness, which he initially claimed resulted from being involved in a motor vehicle collision. Initial ER workup focused on ruling out lifethreatening traumatic injury related to the supposed motor vehicle collision. While on the rehabilitation unit, the patient confessed that he had in fact lost consciousness for close to $24 \mathrm{~h}$ after self-injecting with intravenous heroin.

Vital signs recorded at presentation were as follows: temperature $99.1^{\circ} \mathrm{F}$, pulse 100 beats $/ \mathrm{min}$, blood pressure $120 / 60 \mathrm{mmHg}$, respiratory rate 16 breaths/min, and oxygen saturation of 100 percent on room air. Physical examination showed a tense and tender right gluteal compartment; 0/5 strength on the manual muscle test throughout the right lower limb, hip, knee and ankle; decreased sensation to light touch throughout the right lower limb, including the dorsal and plantar foot; insensate dorsum and plantar right foot; and palpable pedal pulses, $1+$ on the right and 2+ on the left. Initial laboratory findings showed the patient to be in acute renal failure, with laboratory findings of blood urea nitrogen $51 \mathrm{mg} / \mathrm{dL}$, creatinine $4.8 \mathrm{mg} / \mathrm{dL}$, potassium $5.8 \mathrm{mg} / \mathrm{dL}$, and creatine phosphokinase 112,000 IU/L. He also under- went computed tomography per trauma protocol, including that of the spine, pelvis, and leg.

A gluteal CS was suspected, and the patient was taken to the operating room for emergent gluteal fasciotomy. Operative findings included a dusky gluteus medius and a return of blood flow after compartment release. He spent $13 \mathrm{~d}$ in the surgical intensive care unit and required hemodialysis to resolve the rhabdomyolysis-induced acute renal failure. He spent a further $9 \mathrm{~d}$ on the trauma service and underwent electrodiagnostic evaluation (3 wk postinjury), which revealed evidence of acute lumbar 5 to sacral 1 radiculopathy. The needle electromyography results are summarized in the Table. The patient reported resolution of his right buttock pain before being admitted to the inpatient acute rehabilitation unit.

Several days into his rehabilitation course, the patient complained of new pain in his right leg. Physical examination revealed allodynia along the right leg and foot, dry skin, and a side-to-side skin temperature discrepancy with the right warmer compared with the left lower limb. CRPS was suspected. Despite titration of oral analgesics, his pain proved to be severely debilitating and precluded cooperation with therapy. The interventional pain service was consulted. On day 14 of his acute rehabilitation stay, he underwent a trial lumbosacral sympathetic block and continuous lidocaine infusion on days 21 through 23, after which the patient reported a significant reduction in pain and sensitivity such that he was able to resume therapy. He was then successfully transitioned to oral analgesics: gabapentin and tramadol. He was discharged home after $25 \mathrm{~d}$ on the acute rehabilitation unit, ambulating with bilateral crutches at community distances despite a persistent right foot drop.

Table.

Electromyography summary.

\begin{tabular}{|c|c|c|c|c|c|c|c|c|c|}
\hline Muscle $^{*}$ & \multicolumn{5}{|c|}{ Spontaneous } & \multicolumn{3}{|c|}{ Motor Unit Action Potential } & $\begin{array}{c}\text { Recruitment } \\
\text { Pattern }\end{array}$ \\
\hline Tibialis Anterior & $\mathrm{N}$ & $2-3+$ & $2-3+$ & None & None & - & - & - & - \\
\hline Vastus Lateralis & $\mathrm{N}$ & None & None & None & None & $\mathrm{N}$ & $\mathrm{N}$ & $\mathrm{N}$ & $\mathrm{N}$ \\
\hline Gluteus (medial) & $\mathrm{N}$ & $2-3+$ & $2-3+$ & None & None & 一 & - & - & - \\
\hline S1 PSP & $\mathrm{N}$ & $1+$ & $1+$ & None & None & - & - & - & - \\
\hline
\end{tabular}

*All right side.

IA = insertional activity, Fasc = fasciculation, Fib = fibrillation, $\mathrm{HF}=$ high-frequency potentials, $\mathrm{L}=$ lumbar, $\mathrm{N}=$ normal, $\mathrm{PPP}=$ polyphasic potentials, $\mathrm{PSP}=$ progressive supranuclear palsy, PSW = positive sharp waves, $\mathrm{S}=$ sacral. 
On follow-up 1 mo after discharge, the patient's pain remained under control with gabapentin only, he did not show signs of CRPS, and he continued to ambulate with crutches. On this visit, oral consent was obtained to present this case report for publication.

\section{DISCUSSION}

Gluteal CS is a rare event. A literature search yielded fewer than 12 cases in the past 20 yr. The gluteal region is made up of three compartments. The first contains the gluteus maximus, the second the gluteus medius and minimus, and the third the tensor fascia lata [4]. While no major neurovascular structures course through the compartments themselves, an acute rise in pressure may cause damage by compressing the sciatic nerve where it courses between the gluteus maximus and the external rotator complex of the pelvis [5]. A common theme in the clinical histories of the reported cases is prolonged immobilization following drug abuse [6]. Less frequently, surgical positioning has played a role [3].

Early diagnosis is imperative to decrease the likelihood of tissue damage via hypoxemia and compressive forces. In this case, time to ER presentation was delayed by the immobilization itself, as well as the patient's delayed decision to go to the ER. Definitive treatment for CS is urgent fasciotomy. A large incision is made in the muscle fascia to relieve intracompartmental pressure and to restore circulation to the affected tissue or muscle.

Many patients may develop sequelae of CS, especially when diagnosis or treatment is delayed. These sequelae may include CRPS and physical debility. In this case, even though fasciotomy was performed and a dusky gluteus medius had return of color intraoperatively, the sciatic nerve was, in all likelihood, already severely damaged. The clinical findings of objective weakness, muscle atrophy, and sensory loss were consistent with the electrodiagnostic findings suggestive of severe, lumbosacral plexus axonal loss (Table). The finding of lumbar paraspinal positive sharp waves was not consistent with the clinical picture and may have been a false-positive result rather than a true concomitant lumbar radiculopathy [7].

CRPS is a chronic pain condition that has been described by the International Association for the Study of Pain as an array of painful conditions following soft tissue injury, appearing regionally, with pain often exceeding that of the inciting event. It may also be accompanied by significant impairment in motor function [8-9]. CRPS is classified as either type I, also referred to as "reflex sympathetic dystrophy,” or type II, “causalgia,” which develops after confirmed injury to a peripheral nerve. The area affected by CRPS is typically not limited to the distribution of a single peripheral nerve and is associated with evidence of edema and changes in vasomotor and pseudomotor activity. Pain may span an entire limb despite a precipitating injury to only a limited area. CRPS pain may range from moderate to severe and may be described as burning or pins and needles. Objective findings may include edema of the affected limb, discoloration (pale, red, blotchy, or blue), and trophic changes. Changes in skin texture may result in a shiny or thin appearance, while abnormal sweating patterns may also develop. Damage to the microcirculation may cause temperature differences between the affected and unaffected limb. In some cases, CRPS can include changes in nail and hair growth, as well as joint stiffness. Conservative management including oral medications and physical modalities are the mainstay of initial treatment for CRPS. However, in such cases as this where the symptoms do not respond to conservative treatment, one may consider to attempt minimally invasive procedures such as sympathetic ganglion blocks to reduce symptom severity and facilitate function.

Lumbar sympathetic ganglion blocks, such as the block performed in this case, may involve the injection of local anesthetic agents to the lumbar sympathetic chain under fluoroscopic guidance. The lumbar sympathetic ganglia provide sympathetic innervation to the lower limbs. While there is a lack of high-quality evidence for the use of sympathetic blockade for CRPS, it continues to be used in clinical practice in patients whose debilitating pain is refractory to conservative management early in the course of CRPS. While the depth of the controversy surrounding sympathetic blocks for pain stretches beyond the scope of this report, it is important to appreciate that patients with acute CRPS have an increased likelihood of a favorable outcome after sympathetic block compared with those with chronic CRPS [10]. In this case report, we had an acute CRPS diagnosis that was treated rapidly and showed a favorable outcome, similar to the findings of Goebel [10]. In light of treating this condition early and in the setting of mixed evidence regarding efficacy and prognosis, it is difficult to conclude whether this intervention ultimately improved the prognosis of this patient. Further research must be conducted to more fully understand the process of CRPS as well as evaluate and optimize its treatment in refractory cases. 


\section{CONCLUSIONS}

Correct diagnosis, followed by effective management of CRPS, will significantly affect the patient's ability to benefit from physical therapy while recovering in the inpatient rehabilitation setting. Conversely, inadequately treated pain will make participation in therapy more onerous. This was very apparent in this case, in which an otherwise young and motivated individual was reluctant to participate in inpatient therapy sessions because of pain.

The patient's rehabilitation improved markedly after being diagnosed with CRPS type II and receiving a sympathetic lumbosacral plexus block with lidocaine infusion that provided pain relief. After this, he was able to continue with acute rehabilitation. He was discharged with crutches, and his pain was controlled with oral medications.

\section{ACKNOWLEDGMENTS}

\section{Author Contributions:}

Drafting of manuscript: A. Lederman, D. Turk.

Critical revision of manuscript for important intellectual content:

A. Lederman, D. Turk, M. Stern, S. Reddy, A. Howard.

Financial Disclosures: The authors have declared that no competing interests exist.

\section{REFERENCES}

1. Sebastin SJ. Complex regional pain syndrome. Indian J Plast Surg. 2011;44(2):298-307. [PMID:22022040] http://dx.doi.org/10.4103/0970-0358.85351

2. Narayan N, Griffiths M, Patel HD. Gluteal compartment syndrome with severe rhabdomyolysis. BMJ Case Rep. 2013;2013. [PMID:23933861] http://dx.doi.org/10.1136/bcr-2013-010370

3. Golling M, Fonouni H, Mehrabi A, McArthur N, Huber FX. Crush syndrome due to drug-induced compartment syndrome: A rare condition not to be overlooked. Surg Today. 2009;39(7):558-65. [PMID:19562441] http://dx.doi.org/10.1007/s00595-009-3928-6
4. Hayden G, Leung M, Leong J. Gluteal compartment syndrome. ANZ J Surg. 2006;76(7):668-70. [PMID:16813640] http://dx.doi.org/10.1111/j.1445-2197.2006.03797.x

5. Bleicher RJ, Sherman HF, Latenser BA. Bilateral gluteal compartment syndrome. J Trauma. 1997;42(1):118-22. [PMID:9003270] http://dx.doi.org/10.1097/00005373-199701000-00022

6. Adrish M, Duncalf R, Diaz-Fuentes G, Venkatram S. Opioid overdose with gluteal compartment syndrome and acute peripheral neuropathy. Am J Case Rep. 2014;15:22-26. [PMID:24459539] http://dx.doi.org/10.12659/AJCR.889954

7. Date ES, Mar EY, Bugola MR, Teraoka JK. The prevalence of lumbar paraspinal spontaneous activity in asymptomatic subjects. Muscle Nerve. 1996;19(3):350-54.

[PMID:8606700]

http://dx.doi.org/10.1002/(SICI)10974598(199603)19:3<350::AID-MUS11>3.0.CO;2-W

8. Schwarxer A, Maier C. Chapter 33: Complex regional pain syndrome. In: Kopf A, Patel NB, editors. Guide to pain management in low-resource settings. Seattle (WA): IASP 2010.

9. Wasner G, Backonja MM, Baron R. Traumatic neuralgias: complex regional pain syndromes (reflex sympathetic dystrophy and causalgia): clinical characteristics, pathophysiological mechanisms and therapy. Neurol Clin. 1998;16(4): 851-68. [PMID:9767066] http://dx.doi.org/10.1016/S0733-8619(05)70101-8

10. Goebel A. Complex regional pain syndrome in adults. Rheumatology. 2011;50(10):1739-50. [PMID:21712368] http://dx.doi.org/10.1093/rheumatology/ker202

Submitted for publication January 10, 2015. Accepted in revised form July 21, 2015.

This article and any supplementary material should be cited as follows:

Lederman A, Turk D, Howard A, Reddy S, Stern M. Case study: Gluteal compartment syndrome as a cause of lumbosacral radiculoplexopathy and complex regional pain syndrome. J Rehabil Res Dev. 2016;53(4):483-86. http://dx.doi.org/10.1682/JRRD.2015.01.0007

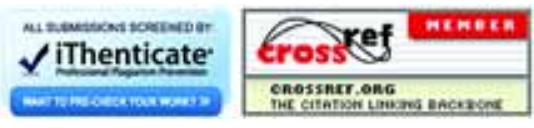

